\title{
A robustness metric integrating spatial and temporal information: application to coral reefs exposed to local and regional disturbances
}

\author{
Andrew R. G. Price ${ }^{1, *}$, Matt J. Keeling ${ }^{1,2}$, Ian N. Stewart ${ }^{2}$ \\ ${ }^{1}$ Ecology \& Epidemiology Group, Department of Biological Sciences, and ${ }^{2}$ Mathematics Institute, University of Warwick, \\ Coventry CV4 7AL, UK
}

\begin{abstract}
Capturing complex ecosystem dynamics through aggregate metrics is challenging, especially against a background of climate instability and local disturbances. Robustness, a key determinant of long-term success or failure, is particularly awkward to distil, because it also conveys many different meanings. Hence, metrics tend to be limited or specialised. Herein we develop an index $(Q)$ which captures one aspect of robustness and, unusually, integrates both spatial and temporal information. $Q$ is defined as the probability that the value of a macroscopic system feature remains above a specified fraction of 'baseline' condition, at a proportion of spatial localities sampled over time. As a test case we analyse coral cover from a long-term survey in Thailand. Sites were subjected to dredging effects in 1986 an 1987 (Sites A and B), elevated sea temperatures and depressed sea levels especially in 1997 and 1998 (Sites A, B and C) following climate related events in the Indian Ocean. Sites A and C have similar $Q$ values, because they experienced similar levels of overall disturbance, albeit different types of disturbance. We show that this metric can also assess specific (e.g. climate related) disturbances. The cut-off value of $Q$ for declaring a system robust is arbitrary. However, our analyses support recent biodiversity studies that used the same coral data set, which suggest remarkable robustness of these reef flats to adverse environmental conditions. Our metric could potentially also quantify change in composition of process oriented features, such as key functional groups, a better proximal determinant of robustness of reefs to hurricanes and other agents of environmental change than coral cover. Probabilistic measures similar to $Q$ could have even wider application, for example to quantify feedback and self-regulation. In a greenhouse world, this behaviour in ecosystems may become increasingly significant.
\end{abstract}

KEY WORDS: Robustness · Spatiotemporal information · Global change $\cdot$ Coral reefs

\section{INTRODUCTION}

Robustness is a key determinant of long-term success or failure in ecosystems and many other systems (Jen 2005a). While the concept shares features in common with stability, it addresses behaviour in a broader class of systems, disturbances and features that are disturbed (Jen 2005b). Ecosystems are increasingly subjected to both acute impacts, such as dredging and oil spillages in marine environments, and chronic disturbances, including climate change (Bellwood et al. 2004, Hughes et al. 2005). Climatic effects extend to bleaching and mass mortality of coral reefs from unusually high sea surface temperatures, for example during El Niño Southern Oscillations (ENSOs) (Price \& Clark 2000, Sheppard \& Rioja-Nieto 2005). Additionally sea-level anomalies, probably linked to ENSOs in the Indian Ocean (Allan et al. 2001), can depress sea level up to $0.3 \mathrm{~m}$ and expose corals to air, with potentially lethal effects (Brown et al. 2002). With the capacity to consider different types of disturbance, robustness could provide a useful framework for characterising effects in ecosystems. 
The scales at which features of ecosystems operate and interact, however, are highly variable (McGlade \& Price 1993, Nyström \& Folke 2001, Hughes et al. 2005, Walker et al. 2005, Webb \& Levin 2005). Hence, their dynamics are often complex and awkward to simplify, although recent progress has been made through meta analysis (Gardner et al. 2003) spatial ecology (Dieckmann et al. 2000, Fortin \& Dale 2005) and analysis of complex temporal series (e.g. Turchin 2003). Robustness, often termed resilience in ecology (Walker et al. 2005, Webb \& Levin 2005), is a particularly difficult concept to synthesise. System complexity aside, the difficulty arises from the diverse and even contradictory meanings ascribed to the concept (Jen 2005a,b, Webb \& Levin 2005); these include, but are not limited to, maintenance of macroscopic system features in the face of a specified assembly of disturbances, selfassembly, self-replication and self-regulation (Webb \& Levin 2005, see also http://discuss.santafe.edu/ robustness). Self-regulation resonates strongly with ecosystem resilience-the capacity to absorb disturbance, reorganise and adapt to change in complex systems with multiple stable states (Gunderson 2000, Nystrom \& Folke 2001, Bellwood et al. 2004).

Despite the intricacies surrounding robustness concepts, aggregate metrics are urgently needed, especially for process-oriented features in marine ecosystems (Hughes et al. 2005). In situations where precise predictions are required, understanding fine-resolution changes through time, space or both may matter most; however, important insights can emerge from distilling system dynamics into simplified representations, for example of robustness (Webb \& Levin 2005). Similarly, in ecology, robustness indices should facilitate regional-scale comparisons across sites and across regions, as input to environmental protection policies at large scales. Yet aggregate measures should be informed by underlying spatio-temporal dynamics.

Here we provide an intuitive approach for characterising robustness, focusing on macroscopic feature persistence and touching on self-regulation. Decomposing raw data into the product of spatial and temporal components defines 'baseline' behaviour and also pinpoints spatio-temporal variability. More importantly, using a probabilistic approach, we collapse irregular data into a single metric of robustness in relation to baseline. As a test case we analyse a macroscopic ecosystem feature (overall coral cover) from a longterm data set for individual species cover derived from multiple localities/transects in Thailand (see Fig. 1). The area has been disturbed by climatic/regional instabilities, dredging and sedimentation. This comprehensive data set is obviously a significant resource for biodiversity analysis, and it has been used to investigate changes in various univariate and multivariate indices (Brown \& Holley 1984, Brown et al. 1990, 2002, Clarke et al. 1993). Our main concern here is to develop a generic approach to the spatio-temporal quantification of robustness. As noted by others (Walker et al. 2005) assessment of systems that are spatially heterogeneous is especially challenging. Although the context here is global change and ecosystem disturbance, the same or similar methodology could potentially characterise other systems with simultaneously explicit temporal and spatial data.

\section{MATERIALS AND METHODS}

Background: robustness concepts. Wagner's (2005) recent major work highlights the need to be explicit about robustness of what (system feature) to what (specific disturbance). In marine ecology, in particular, knowledge of all disturbances will often be incomplete. The present study quantifies robustness of coral cover to dredging and ENSO/sea-level disturbances, and possibly to others unknown, including natural change. Wagner (2005) focuses on genetic robustness, with the author pointing out that a review of robustness to environmental and other non-genetic changes would be daunting indeed. In our 'Introduction', we briefly mentioned relationships between robustness, resilience and stability. As indicated, resilience is a term for robustness often used in ecology, a point also made by Wagner (2005). The 'resilience alliance' (Walker et al. 2004, see also www.resalliance.org/ 1.php) suggests that for any organisational scale, resilience of a system has 4 key aspects: latitude, the greatest change possible before losing capacity to recover; resistance, ease or difficulty of altering it; precariousness, current proximity to a limit or threshold; and panarchy, influences from states and dynamics at scales above and below that of the system feature(s) of interest, because of cross-scale interactions (www. resalliance.org/1.php). Of significance is that resistance and resilience are among the 45 or more variations in meaning of stability (Lehman \& Tilman 2000). Several reviews and studies on resilience/robustness are available (e.g. Peterson et al. 1998, Gunderson \& Pritchard 2002, Walker et al. 2004, Hughes et al. 2005). Herein, we use the term robustness, partly because it is used in cross-disciplinary contexts and because our metric has application both within and potentially beyond marine ecology.

Study area. The study area comprised 3 sites (A, B and C) on shallow reef flats up to $200 \mathrm{~m}$ wide on the southeast tip of Ko Phuket, Thailand (Fig. 1a). It had been subjected to several disturbances, with dredging the main local perturbation, beginning on 21 October 1986 and continuing to mid-July 1987 (Brown et al. 2002). Al- 
a

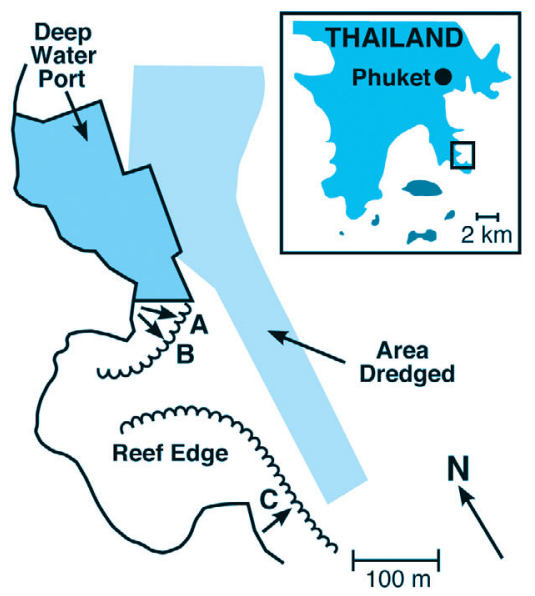

C

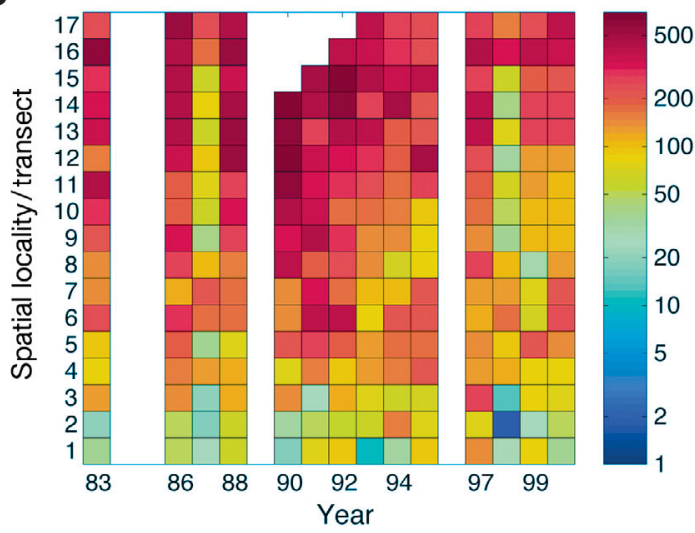

b

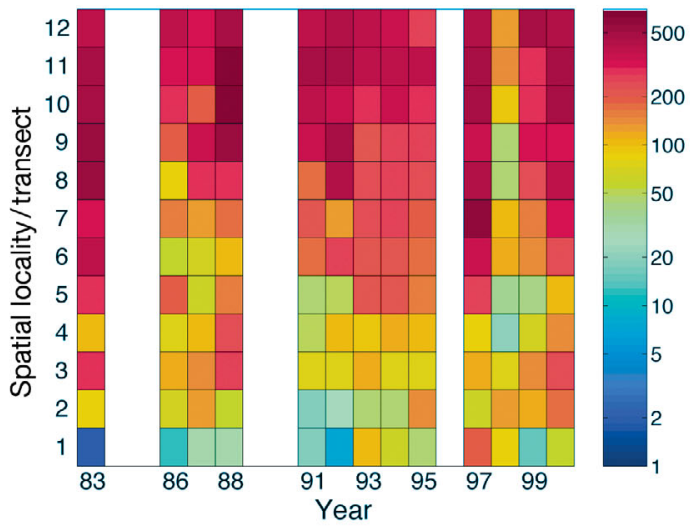

d

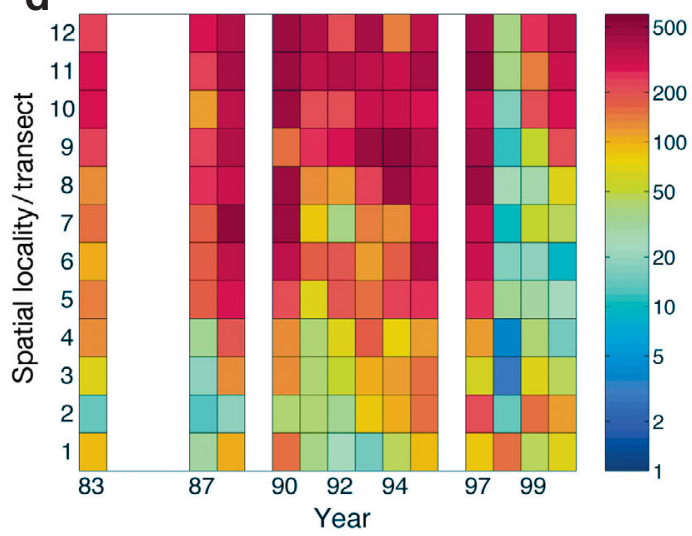

Fig. 1. Case study sites at Ko Phuket, Thailand, and time series for coral cover data at multiple spatial localities/transects (12 at Sites A and C, 17 at Site B) used for computation of robustness. (a) Location of Sites A and B (close to dredging and sedimentation) and Site C (reference area); (b-d) raw coral cover data $(\mathrm{cm})$ for each $10 \mathrm{~m}$ transect at Sites A, B and C, respectively. Total coral cover is colour coded on right-hand scale

though dredging continued for 9 mo, fallout of fine sediments was confined to the period between October 1986 and January 1987. Over 1.3 million $\mathrm{m}^{3}$ of sediment were excavated and transported to the deep-water port. Site A had greatest exposure to sediments, Site B was expected to be somewhat less affected, while Site $\mathrm{C}$ received relatively low levels of sediment (Brown et al. 1990).

Regional (climatic) perturbations have involved elevated sea temperatures in 1991, 1995, 1997 and 1998 at all sites, and anomalously low tides $(>-30 \mathrm{~cm})$ in 1994 , 1997 and 1998 at all sites (Brown et al. 2002). The latter are associated with Indian Ocean dipole events arising from ocean-atmosphere-land interactions that have occurred in $16 \mathrm{yr}$ between 1950 and 1998 (Chambers et al. 1999, Brown et al. 2002). Many corals bleached during elevated sea temperatures in 1991 and 1995, but no bleaching was recorded in 1997. Very limited bleaching occurred in 1998 although sea temperatures were higher than previous events in 1991 and 1995 (Brown et al. 2002). Being intertidal, corals at the study sites are periodically exposed and therefore potentially highly vulnerable to changes in sea level and climatic disturbances.

Data set. Coral cover data originate from monitoring studies sampled over periods of either $13 \mathrm{yr}$ (Sites A and C) or 14 yr (Site B, with an additional sample in 1986) between 1983 and 2000. Permanent transects were placed parallel to the shore at $10 \mathrm{~m}$ intervals from the inner reef flat to the outer reef edge. Sites A and C comprised $12 \times 10 \mathrm{~m}$ transects, and Site B $17 \times 10 \mathrm{~m}$ transects. Transects (Fig. 1a) provided a measure of coral cover (Fig. 1c-d) by plotless line technique (Brown et al. 2002), whereby cover (abundance) is the linear extent $(\mathrm{cm})$ of coral summed for every species. These same monitoring sites have been used to study the effects of local and regional disturbances on biodiversity and assemblage, but utilising species-level data (Brown et al. 1990, 2002, Clarke et al. 1993).

Analysis. For each of the 3 sites $(\mathrm{A}, \mathrm{B}$ and $\mathrm{C})$, we have a matrix of observed data $\left(D_{l, t}\right)$, which is the measured coral cover in transect (location) $l$ in Year $t$. We propose to decompose these data into 4 constituent 
components reflecting the effects of spatial location (transect number), temporal forcing (year), population dynamics and noise - this is performed separately for the 3 sites. Mathematically the dynamics for each transect, reflecting spatio-temporal variability, are captured as follows:

$$
D_{l, t}=S_{1} \times T_{t} \times F\left(D_{1, t-1}, D_{1, t-2}, D_{1, t-3} \ldots\right) \times R_{l t}
$$

We assume the data to be determined by 4 major components: $S_{l}$ is the spatial component, reflecting the aggregate 'quality' (here coral cover) at Location (Transect) $l_{i} T_{t}$ is the temporal component, which accounts for variability between years across all locations; $F\left(D_{1, t-1}, D_{1, t-2}, D_{1, t-3} \ldots\right)$, a dynamic component, is a general function of the lagged data for each location that describes the underlying deterministic processes and thus captures temporal autocorrelations within the data (Appendix 1); $R_{1 t}$ is the residual component, which accounts for all variation not captured by the other 3 deterministic components. The values of $S$ and $T$ (and the underlying deterministic function, $F$ ) are found by minimising the mean square of the logged residuals (Appendix 1).

Interactions between components are assumed to be multiplicative, such that a change in temporal conditions (on average) results in the same percentage change at all localities. Parameters are scaled, such that the geometric average value of $T_{t}$ is 1 . This represents 'baseline', with quantities above or below corresponding to conditions likely to enhance or depress abundance observations.

We take our aggregate metric of robustness as the probability of a set of observations on coral cover (or other system feature) at all or a proportion of spatial localities exceeding a specified fraction $(p)$, of the expected 'baseline' value across $k$ years. Thus, this measure intuitively integrates spatial and temporal variability into an index whose values range from 0 to 1. Based on the coral reef data, we define the index of robustness $(Q)$ as:

$$
Q=Q_{k p}=\left\{\int_{0}^{\infty} \operatorname{Pr}(T=s)\left[\operatorname{Pr}\left(R>\frac{p}{s}\right)\right]^{n} \mathrm{~d} s\right\}^{k}
$$

where we have assumed that the temporal variation $(T)$ and the residuals $(R)$ that are observed reflect realisations from some probabilistic distribution. This equation for $Q$ calculates the probability that the product of temporal and residual variation is greater than $p$ in $n$ different locations over $k$ years; where $n$ is the chosen number (some or all) of spatial transects, $T$ is temporal variation, $p$ is the chosen fraction of the baseline value, $R$ is the residuals and $k$ is the number of consecutive time points. Values of $Q$ are derived from a non-parametric fit to the observed cumulative probability distribution of $T$, fitted with a cubic spline and by assuming a log-normal distribution for $R$. (However, results are robust to different smoothing methodologies.) Eq. (2) applies only when there are no temporal autocorrelations in the data. When autocorrelations do occur, however, an index analogous to $Q$ can still be defined and computed from data although its formal expression is complex (which falls beyond the scope of this study). Values of $Q$ are calculated for each of the sites (A, B and C) and the significance of differences between the values is assessed by bootstrapping (see Appendix 1).

\section{RESULTS AND DISCUSSION}

\section{Spatio-temporal variability and baseline}

Analysis of the raw coral cover data (Fig. 1b-d) and the data scaled to baseline (Fig. 2) reveals fine-scale spatio-temporal variability at all 3 sites. (Actual values ranged from 2 to $722 \mathrm{~cm}$ per $10 \mathrm{~m}$ transect, i.e. a maximum of $>70 \%$ coral cover.) The downturns in 1986 and 1987 at Site A, and single (but bigger) dip in 1987 nearby at Site B, may partly reflect the heavy exposure of these sites to dredging and sedimentation disturbance (see 'Materials and methods'). However, the subsequent regional perturbation in 1998 was far greater; paradoxically effects were most marked at Site C. This drop in temporal components at all 3 sites probably reflects the effects of low sea level (ca. $-30 \mathrm{~cm}$ ) arising from the Indian Ocean dipole that year (Fig. 2). Despite the strong El Niño (Fig. 2) and relatively high sea temperatures that year, coral bleaching was actually limited (see 'Materials and methods'). Using multivariate measures, other workers (Brown et al. 2002) also reported marked downturns at Sites A and B in 1986 and 1987 from dredging and at all sites in 1998 from negative sea-level anomalies. However, the Indian Ocean dipole is known to be linked to ENSOs, albeit in complex ways (Allan et al. 2001).

\section{Robustness metric $(Q)$ computations}

Values of $Q$ are computed for the 3 coral cover data sets scaled in relation to baseline (Fig. 2). With $Q$, set as the probability that all spatial localities sampled are greater than $50 \%$ of baseline for any given year $(n=12$ for Sites $\mathrm{A}$ and $\mathrm{B}, n=17$ for Site $\mathrm{B}, p=1 / 2, k=1$ ), we obtained the following results: $Q=0.37$ (Site A), $Q=$ 0.44 (Site B) and $Q=0.38$ (Site C). Differences are not statistically significant $(p \geq 0.05)$. Sites $A$ and $C$ have very similar $Q$ values, because they experienced similar levels of overall disturbance, albeit different types of disturbance (i.e. heavy dredging sedimentation at 

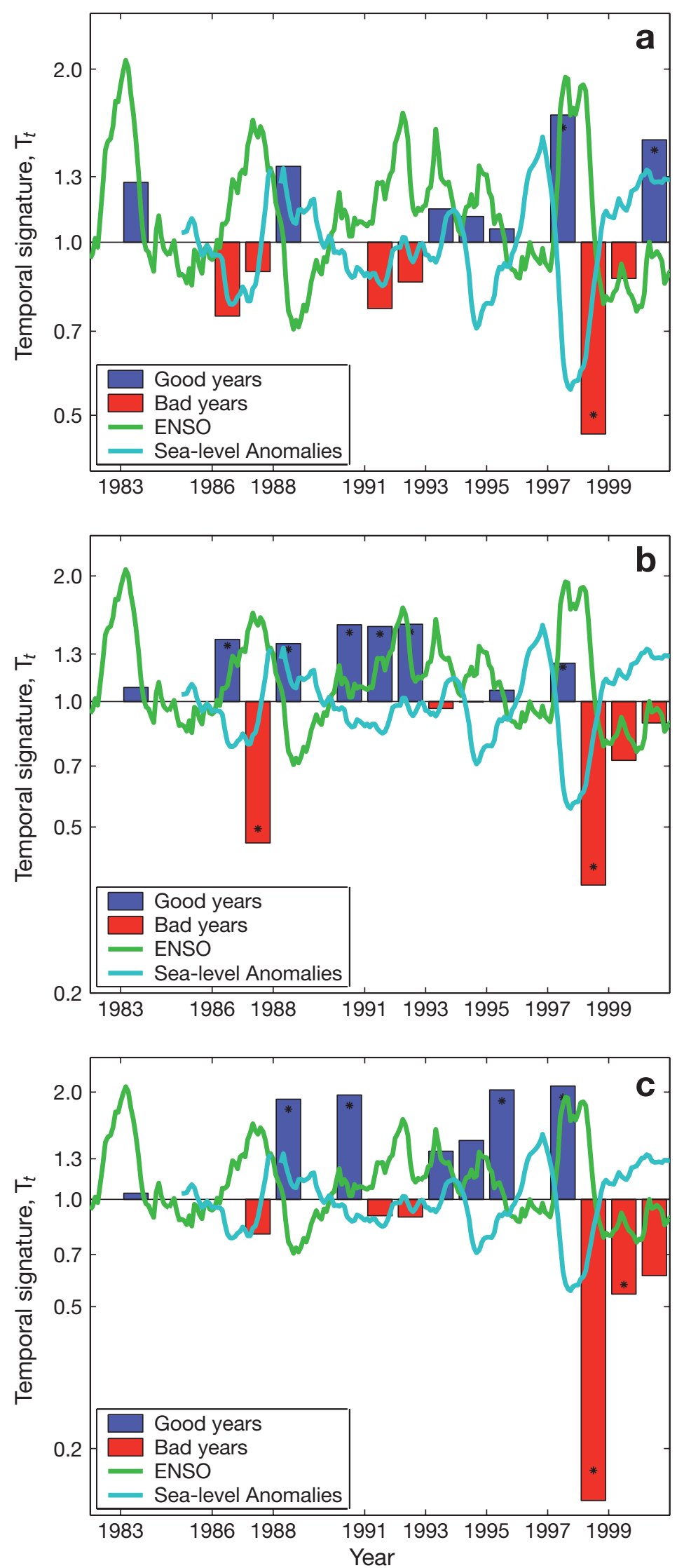

Site A, but low to moderate climatic/regional disturbances; low dredging sedimentation, but marked climatic/regional disturbances at Site C). Overall disturbance at Site B was slightly less, reflected by the higher value of $Q$.

Of course, chosen values for $n$ and $p$ (Eq. 2) and duration of the time series used for $Q$ are arbitrary. If we fix the temporal component to be that experienced in 1998 (instead of using the distribution from all years), but retain the baseline derived from the whole time series (Fig. 2) and with $n$ and $p$ unaltered, the results are very different. $Q$ drops markedly and differences become highly significant ( $\mathrm{p} \leq 0.01)$ : $Q=6 \times 10^{-5}$ (Site A), $Q=1 \times 10^{-10}$ (Site B) and $Q=2 \times 10^{-20}$ (Site C). Now Site C (not Site A) has by far the lowest value (Fig. 2c), because 1998 was highly atypical and severe, especially at that site. This example confirms that Site $\mathrm{C}$ was affected by disturbance but, as mentioned, that year it was regional/climatic. These 2 examples demonstrate that our metric can tell us about both robustness to disturbance in general and to specific disturbances.

$Q$ is computed only for a single time point $(k=1)$, both in this example and in the earlier one, resulting from calculation of probability in any (single) year. However, the equation can accommodate multiple consecutive points $(k>1)$, such as the years 1998, 1999 and 2000).

As for any scalar metric, tradeoffs may arise from different averaging processes, by choosing differing values for the fraction of the baseline value $(p)$, the number of consecutive time points $(k)$ and the number of spatial localities $(n)$. For example, the probability that the value of a macroscopic feature, such as coral cover, exceeds a high 'threshold' (fraction of baseline) value for 1 or a few time points may be equivalent to the probability of it exceeding a lower 'threshold' value but for a greater number of

Fig. 2. Temporal component/signature $\left(T_{t}\right)$ extracted from coral cover data, to differentiate between good and bad years, and regional/climatic events, showing coral signatures for Sites A (a), B (b) and C (c). Baseline is scaled such that average $\mathrm{T}_{t}=1$, which is used in estimates of robustness index, $Q$ (see 'Materials and methods'); values significantly different from 1 are marked with an asterisk. The relative scaled magnitudes of El-Niño Southern Oscillations (ENSOs) and sea-level anomalies from 1983 to 1999 for Ko Taphao Noi tide station $(2.5 \mathrm{~km}$ from our study sites) are superimposed showing their deviation from the mean (ENSO values range from -2.25 to 3.16, sea-level anomalies from 2061 to $2306 \mathrm{~mm}$ ) 
time points, although conceptually these are very different properties. Similarly, abundance itself depends not only on the chosen fraction of the baseline value, but also on the proportion of spatial localities fulfilling this. Hence, 'moderate' abundance might arise from the probability that coral cover exceeds $50 \%$ of the baseline value for all spatial localities, or that it exceeds $75 \%$ of baseline at fewer localities. Clearly, this sort of interplay is hypothetical and idealised, for in real systems spatial/temporal interactions are complex (Walker et al. 2005). Nevertheless the sorts of tradeoffs that potentially influence $Q$ would seem intuitive, resonating with, for example, net effects of oil spills. Impact from an extensive, short-term oil spill may be similar, qualitatively, to that from a smaller but longerlasting spill. Indeed, for damage compensation, 'habitat equivalency analysis' combines space and time, using (discounted) $\mathrm{km}^{2}$ yr as currency (Dunford et al. 2004).

The cut-off value of $Q$ for declaring something robust, or fragile, is rather arbitrary. Nevertheless, based on recovery from local and regional perturbations (Fig. 2), reef flats in Thailand appear remarkably robust; in terms of effects of disturbance on coral cover, history seems unimportant (Fig. 2). Whatever the stresses, coral quickly re-grows, confirming immunity to whole-colony mortality (Brown et al. 2002). Being shallow and semi-intertidal, these reef flats are naturally stressful environments. Through evolutionary processes, the roughly 30 corals present (Brown \& Holley 1984) are a small but 'robust' subset of the 206 species known for the region (Sheppard 1998).

The amount of data required before $Q$ becomes 'reliable' is not straightforward; it depends very much on the data set. In our study, if 1998 data on coral cover are omitted there is a big effect, whereas loss of 1983 data would produce a much less marked effect.

Another aspect of robustness, also measurable by $Q$, is effects of disturbance on change in composition of features (Jen 2005b), for example of different functional herbivore groups (bioeroders, scrapers and grazers)-a better proximal determinant of vulnerability and robustness or resilience in reefs than coral cover (Bellwood et al. 2004). Herbivory is one of several factors that can cause reefs to flip between alternate states (Walker et al. 2005) and strongly influences reef condition (e.g. Nyström et al. 2000, Bellwood et al. 2004, Hughes et al. 2005). Development of new metrics applied to essential processes has been recently highlighted as an urgent priority for better stewardship of marine resilience/robustness (Hughes et al. 2005). This represents a shift away from a 'taxonomic' approach and conservation of targeted species towards functional groups and ecological roles (Hughes et al. 2005). Distillation of robustness using spatio-temporal metrics, such as $Q$, may facilitate this, particularly if applied to process oriented system features rather than merely coral cover.

As mentioned, $Q$ or a similar methodology could be used to characterise other systems with simultaneously explicit temporal and spatial data. Many fisheries today are being managed relative to targets, with the aim to keep populations above some defined threshold level (e.g. a $35 \%$ spawning potential ratio to avoid recruitment overfishing). This metric could potentially provide a valuable means of assessing the performance of fishery managers in achieving such targets.

Application of probabilistic measures, similar to ours, could potentially extend to other dimensions of robustness, even outside ecology. In fact, a version of $Q$ that is not spatially explicit would simply measure tendency of a system feature to depart from 'baseline' in the face of some perturbation(s). In both biological and engineering systems this is the essence of negative feedback, or self-regulation. However, robustness is multifaceted (Jen 2005a,b) and it would be impossible for variants of $Q$, and probably any other scalar index, to capture many system complexities (Walker et al. 2004). Hence, we see our approach as complementary to frameworks such as highly optimised tolerance (HOT; Carlson \& Doyle 2002). In engineering, physics and biological systems HOT captures the robust-yetfragile paradox of complex systems. Interestingly, our computations of $Q$ for corals in Thailand reveal variations in robustness to climatic and other disturbances over remarkably short spatial scales $(<0.5 \mathrm{~km})$.

We emphasise that aggregate (and other) statistics require careful interpretation. Use of only recent time series can result in failure to detect deteriorating condition, as studies of Caribbean coral reefs demonstrate (Gardner et al. 2000). There, major disturbance began following an earlier phase change from reef coral to fleshy algal dominance. Without comparative data for previous decades, present reef condition might seem normal, reflecting the 'shifting baseline syndrome' (Sheppard 1995, Jackson 1997, Bellwood et al. 2004). Also the predictive power of our index is unclear, in common with indices of sustainability, which rely on past data. It may be possible to say something has been sustainable and presently appears so. However, because many systems are dynamic, claims that something will be sustainable in the future can be unwarranted (Milner Gulland \& Akçakaya 2001). Ecosystems and non-living systems often behave unpredictably.

Understanding the importance of ecosystem robustness and ability to measure it using effective metrics may become increasingly significant in a greenhouse world. In the Indian Ocean much attention has focused on rising sea temperatures, particularly in association with the 1998 ENSO which caused widespread 
mortality (Sheppard \& Rioja-Neto 2005). In the Caribbean the catastrophic switch of many reefs into algal-dominated systems has been ascribed largely to other impacts (Gardner et al. 2003). Yet the observable increase in sea temperatures in this region (Sheppard \& Rioja-Nieto 2005) could also become increasingly important to the survival of coral communities and their capacity to recover (Knowlton 2001).

Acknowledgements. We thank Barbara Brown for provision of the coral data sets and Charles Sheppard and anonymous reviewers for helpful critiques of early drafts of this paper. We also thank Terry Hughes and others at the Symposium on Coral Reef Conservation in London on 16-17 December 2004 for their helpful discussions. We are grateful to Sam Mason for assistance with graphics. This work was supported by the University of Warwick.

\section{LITERATURE CITED}

Allan RJ, Chambers D, Drosdowsky W, Hendon H and 5 others (2001) Is there an Indian Ocean dipole, and is it independent of the El Niño-Southern Oscillation? Clim Variability Predictability (CLIVAR) Exchanges 6:18-22

Bellwood DR, Hughes TP, Nyström M (2004) Confronting the coral reef crisis. Nature 429:827-833

Brown BE, Holley MC (1984) Coral assemblages of intertidal reef flats at Ko Phuket, Thailand. Res Bull Phuket Mar Biol Cent 30:1-10

Brown BE, Le Tissier MDA, Scoffin TP, Tudhope AW (1990) Evaluation of the environmental impact of dredging on intertidal corals at Ko Phuket, Thailand, using ecological and physiological parameters. Mar Ecol Prog Ser 65: 273-281

Brown BE, Clarke KR, Warwick RM (2002) Serial patterns of biodiversity change in corals across shallow reef flats in Ko Phuket, Thailand, due to the effects of local (sedimentation) and regional (climatic) perturbations. Mar Biol 141: 24-29

Carlson JM, Doyle J (2002) Complexity and robustness. Proc Natl Acad Sci USA 99(Suppl 1):2538-2545

Chambers DP, Tapley BD, Stewart RH (1999) Anomalous warming of the Indian Ocean coincident with El Nino. J Geophys Res 104:3035-3047

Clarke KR, Warwick RM, Brown BE (1993) An index showing breakdown of seriation related to disturbance, in a coralreef assemblage. Mar Ecol Prog Ser 102:153-160

Dieckmann U, Law R, Metz JAJ (2000) The geometry of ecological interactions: simplifying spatial complexity. Cambridge University Press, Cambridge

Dunford RW, Ginn TC, Desvousges WH (2004) The use of habitat equivalency analysis in natural resource damage assessments. Ecol Econ 48:49-70

Fortin MJ, Dale MRT (2005) Spatial analysis: a guide for ecologists. Cambridge University Press, Cambridge

Gardner TA, Cote IM, Gill JA, Grant A, Watkinson AR (2003) Long-term region-wide declines in Caribbean corals. Science 301:958-960
Gunderson LH (2000) Ecological resilience: in theory and application. Annu Rev Ecol Syst 31:425-439

Gunderson LH, Pritchard LH (2002) Resilience and the behavior of large scale systems. Island Press, New York

Hughes TP, Bellwood DR, Folke C, Steneck RS, Wilson J (2005) New paradigms for supporting the resilience of marine ecosystems. Trends Ecol Evol 20:380-386

Jackson JRC (1997) Reefs since Columbus. Coral Reefs 16(Suppl):S23-S32

Jen E (2005a) Introduction. In: Jen E (ed) Robust design: a repertoire of biological, ecological, and engineering case studies. Oxford University Press, Oxford, p 1-6

Jen E (2005b) Stable or robust? In: Jen E (ed) Robust design: a repertoire of biological, ecological, and engineering case studies. Oxford University Press, Oxford, p 7-20

Knowlton N (2001) The future of coral reefs. Proc Natl Acad Sci USA 98:5419-5425

Lehman CL, Tilman D (2000) Biodiversity, stability, and productivity in competitive communities. Am Nat 156:534-552

McGlade JM, Price ARG (1993) Multidisciplinary modelling: an overview and practical implications for the governance of the Gulf region. Mar Pollut Bull 27:361-375

Milner Gulland, EJ, Akçakaya HR (2001) Sustainability indices for exploited populations under uncertainty. Trends Ecol Evol 16:686-692

Nyström M, Folke C (2001) Spatial resilience of coral reefs. Ecosystems 4:406-417

Nyström M, Folke C, Moberg F (2000) Coral reef disturbance and resilience in a human-dominated environment. Trends Ecol Evol 16:413-417

Peterson GD, Cumming GS, Holling CS (1998) Ecological resilience, biodiversity, and scale. Ecosystems 1:6-18

Price ARG, Clark S (2000) The Maldives. In: Sheppard CRC (ed) Seas at the millennium, Vol II. Elsevier, Amsterdam, p 199-219

Sheppard CRC (1995) The shifting baseline syndrome. Mar Pollut Bull 30:766-767

Sheppard CRC (1998) Biodiversity patterns in Indian Ocean corals, and effects of taxonomic error in data. Biodivers Conserv 7:847-868

Sheppard CRC, Rioja-Nieto R (2005) Sea surface temperature 1871-2099 in 38 cells in the Caribbean region. Mar Environ Res 60:389-396

Turchin P (2003) Complex population dynamics: a theoretical/ empirical synthesis. Princeton University Press, Princeton, NJ

Wagner A (2005) Robustness and evolvability in living systems. Princeton University Press, Princeton, NJ

Walker B, Holling CS, Carpenter SR, Kinzig A (2004) Resilience, adaptability and transformability in socialecological systems. Ecology and Society 9(2):5 available at: http://www.ecologyandsociety.org/vol9/iss2/art5

Walker B, Petersen G, Anderies JM, Kinzig A, Carpenter S (2005) Robustness in ecosystems. In: Jen E (ed) Robust design: a repertoire of biological, ecological, and engineering case studies. Oxford University Press, Oxford, p 173-189

Webb CT, Levin SA (2005) Cross-system perspectives on the ecology and evolution of resilience. In: Jen E (ed) Robust design: a repertoire of biological, ecological, and engineering case studies. Oxford University Press, Oxford, p 151-172 
Appendix 1. Additional information on analysis

As indicated in 'Materials and methods', spatio-temporal variability in coral cover data is decomposed into 4 major components (Eq. 1). One component is a dynamic component, $F\left(D_{1, t-1}, D_{1, t-2}, D_{1, t-3} \ldots\right)$. It is a general function of the previous data for each location, to describe dynamic changes due to population processes, such as density dependence. The signature of such population processes is temporal autocorrelations within the data. Fig. A1 shows that in our study there are no autocorrelations and therefore the dynamic component can be ignored and set to 1. $R_{l t}$ is the residual component. (Other times lags, i.e. of more than $1 \mathrm{yr}$, showed similar independence.) Fig. A2 demonstrates the residuals' log-normal distribution about a mean of 1 and minimal correlation with either the spatial $(S)$ or temporal $(T)$ components. This confirms that the residuals are independent of the other components.

To determine which values of $T$ are statistically significant, we generated 10000 resamplings of the data, whereby the temporal data for each transect were randomly permuted. For each of these resamplings, appropriate values of $S$ and $T$ were calculated; the values of $T$ from the observed data were considered significant if they lay outside the 95 percentiles of the resampled data.

To assess the difference between $Q$ values at each of the 3 sites, we again used a boot-strapping technique. Surrogate data were generated from the same $S$ and $T$ values, but with the residuals chosen at random from the appropriate distribution. New $S$ and $T$ values could then be calculated for each set of surrogate set-these may be different from the original values due to the residuals chosen - new $Q$ values could then be computed.

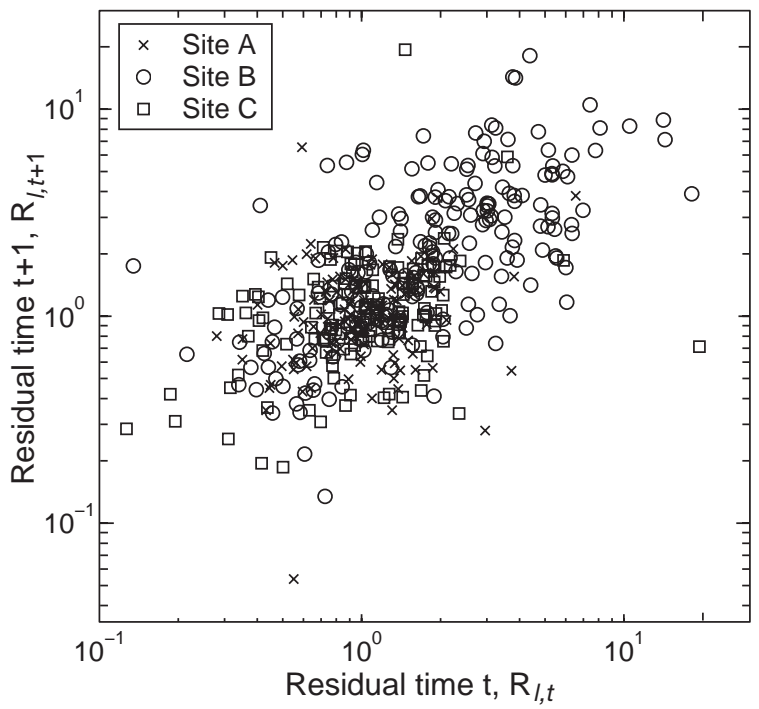

Fig. A1. Temporal autocorrelations in coral cover data. Driven by the underlying dynamics, these are a general function of the previous year's data for Sites A to C. Here the dynamic component $F\left(D_{1, t-1}, D_{1, t-2}, D_{1, t-3} \ldots\right) \approx 1$
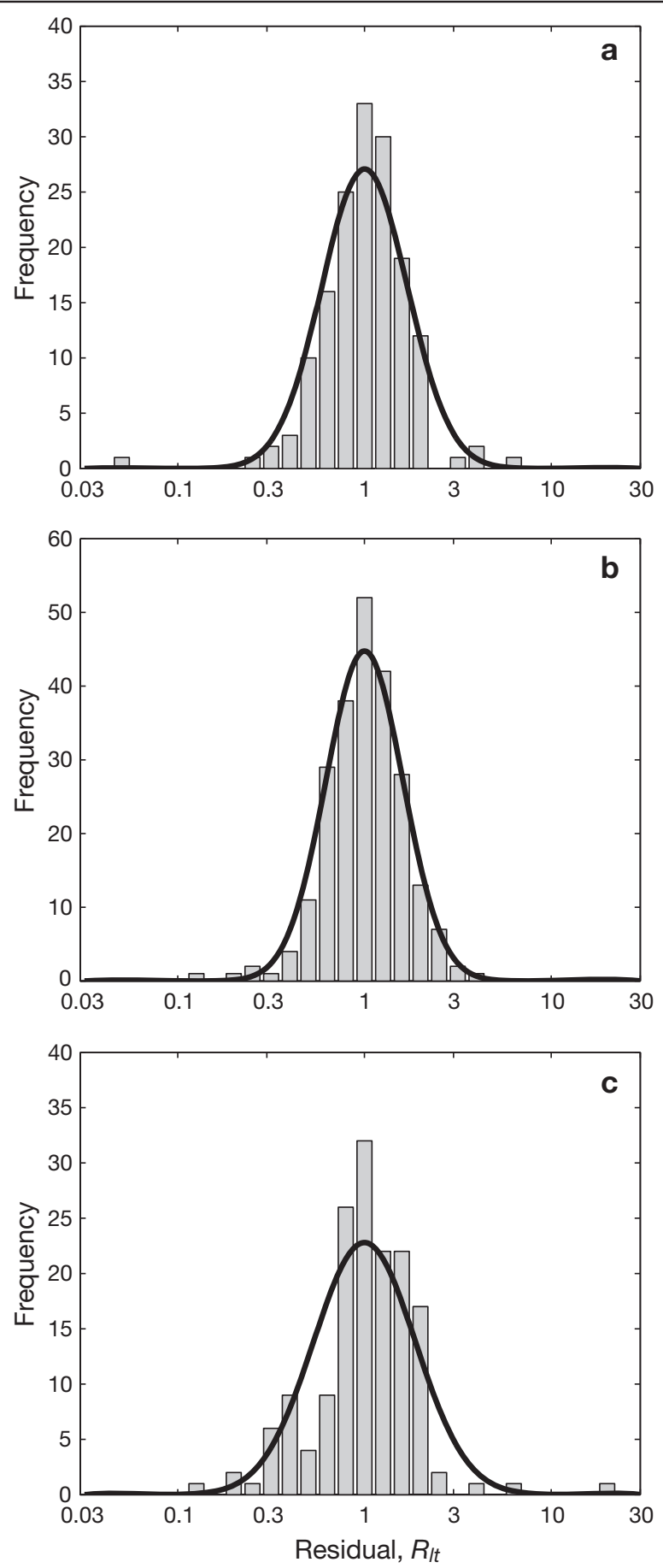

Fig. A2. Residuals or error, $R_{l t}$ associated with decomposition of coral cover data into spatial and temporal components for Site A (a), B (b) and C (c). Log-normal distribution with the same variance structure is shown by continuous line 